\title{
A Patient Portal Intervention for Menopause Knowledge and Shared Decision-Making
}

\author{
Stuti Dang, MD, MPH, ${ }^{1,2}$ Berry Thavalathil, MBBS, ${ }^{3}$ Diana Ruiz, $\mathrm{RN},{ }^{4}$ Carlos Gómez-Orozco, BS, ${ }^{2}$ \\ Orlando Gómez-Marín, $\mathrm{PhD},{ }^{5}$ and Silvina Levis, $\mathrm{MD}^{1,2}$
}

\begin{abstract}
Background: Menopause is a time often fraught with changes and symptoms, which may require difficult choices and decision-making. During this period, women would benefit from a better understanding and indepth discussions with providers regarding menopause, associated conditions, and appropriate therapy. Patient portals offer a potential means to improve knowledge and shared decision-making (SDM) about menopause. Materials and Methods: This protocol article explores the feasibility of using the secure messaging (SM) function of the Veterans Affairs (VA) Patient Portal, "My HealtheVet" to implement an educational intervention and measure its impact on knowledge and SDM in the management of menopause.

Results: This is a quality improvement pilot study in which women veterans of menopausal age in the Miami VA are offered an educational intervention via a patient portal, while women veterans in two neighboring VA facilities are not. Intervention participants receive weekly SMs with information on menopause symptoms, and treatment. After 6-months, all participants are surveyed on menopause knowledge, SDM, and satisfaction with the program.

Conclusion: This study is among the first to assess the impact of an innovative patient portal intervention to improve knowledge and SDM between patients and providers regarding menopause. If successful, our program will add to the "meaningful use" of patient portals and offer a scalable and timely resource for SDM about menopause.
\end{abstract}

Keywords: shared decision-making, patient portals, menopause

\section{Introduction}

$\mathbf{T}$ HE MOST FREQUENT menopausal symptoms include hot flashes, night sweats, insomnia, mood instability, and vaginal dryness, which are often associated with a decrease in quality of life (QoL). ${ }^{1,2}$ While options are available to ease these symptoms, many women do not use them because they may have insufficient knowledge regarding menopause and available treatment options, or are unclear how to choose among these options. ${ }^{3,4}$ Moreover, health care providers frequently neglect to screen and identify symptomatic menopausal women, and often fail to address and treat menopausal symptoms in their patients. ${ }^{5}$ Menopause symptom treatment would benefit from shared decision-making (SDM) as it is a sensitive topic warranting individual case-by-case consultation to evaluate risks and benefits of the various treatment options for each patient. ${ }^{6}$ SDM involves sharing the best available evidence between patients and providers and supporting patients in the process of weighing options and making informed health or treatment choices. ${ }^{7}$ The SDM process is facilitated by patients' understanding of their diagnoses and available treatment options which "increases people's involvement and improves knowledge and realistic perception of outcomes." 8

Based on focus groups conducted in our earlier work, women veterans have many unanswered questions regarding menopause symptoms and management. ${ }^{9}$ Women in this age group often have conflicting work or family responsibilities, making it difficult to schedule health care visits. ${ }^{10}$ Therefore, a need exists for developing innovative

\footnotetext{
${ }^{1}$ Geriatric Research, Education and Clinical Center (11 GRC), Miami Veterans Affairs Healthcare System, Miami, Florida

${ }^{2}$ Department of Medicine, University of Miami Miller School of Medicine, Miami, Florida.

${ }^{3}$ South Florida Veterans Affairs Foundation for Research and Education Miami, Florida.

${ }^{4}$ Research Service, Miami VA Healthcare System, Miami, Florida.

${ }^{5}$ Division of Epidemiology and Public Health Science, University of Miami Miller School of Medicine, Miami, Florida.
} 
educational strategies for women seeking menopause health information. $^{11}$

As defined by the Office of the National Coordinator for Health Information Technology, patient portals are secure online websites that give patients convenient 24-hour access to personal health information from anywhere with an Internet connection. ${ }^{12}$ After entering a username and password, most portals typically enable patients to also exchange secure messages (SM) with their health care team, schedule appointments, or request prescription refills. ${ }^{13}$ Patient portals offer a promising approach to educate patients and have been shown to improve patient knowledge and outcomes in other chronic conditions such as heart failure ${ }^{14}$ and diabetes. ${ }^{15}$ However, little work has been done to explore how patient portals can be used to improve SDM. ${ }^{16,17}$

A recent systematic review ${ }^{18}$ showed that there were few reported patient portal interventions which supported SDM, and even fewer which also measured impact on SDM. The review reported that of 1369 articles, only five studies demonstrated improved clinical outcomes and SDM among patients with use of patient portals aiding SDM. ${ }^{19-25}$ However, none of these five studies were regarding menopause.

In this protocol article, we describe the rationale and protocol of a trial that examines the feasibility of an educational intervention using the Veterans Affairs (VA) patient portal, My HealtheVet (MHV), to specifically assess its impact on knowledge and SDM about menopause.

\section{Materials and Methods}

\section{Overview}

"My HealtheVet to Enable And Negotiate for Shared decision making" or MEANS is a nonrandomized quality improvement pilot study. We will identify all female veterans of perimenopausal and menopausal age (45-60 years) at three VA Healthcare Systems (VAHS). We will offer a 6-month educational intervention using a patient portal to women in one VAHS only, the intervention site. We then will compare the impact of the program on patients at the intervention VAHS to unidentified women veterans of the same age group at two nearby VAHS, the comparison sites, who will continue to receive usual care and will not receive the intervention, as well as to a local comparison group at the intervention site. Outcomes will be assessed before and after the intervention among women in both the intervention and comparison groups, and will be compared within and between intervention and comparison groups. The primary outcome will be change in self-reported menopause knowledge. The secondary outcomes are patient perception of SDM and rates of diagnosis and management of menopause symptoms. We will also conduct a program evaluation to assess satisfaction with various aspects of the program.

Our primary hypothesis is that at the end of the 6-month intervention, patients in the intervention group will have greater improvement in menopause knowledge scores than the comparison groups. We propose that increased patient knowledge and awareness of menopause will lead to increased patient-initiated conversations and SDM regarding menopause during provider visits at the intervention site. Thus, our secondary hypothesis is that after the 6-month intervention, a greater proportion of patients in the intervention group, compared to baseline and to the comparison groups, will have a SDM conversation regarding menopause with their provider. This will be evidenced by an associated increase in number of women with a diagnosis of menopause or beginning medications for menopause symptoms.

The study was reviewed and approved by the Miami VAHS Institutional Review Board and determined to not require documentation of informed consent since it is a quality improvement educational intervention.

\section{Conceptual design}

The guiding framework for this study is a modified "Three Talk Model of Shared Decision Making for Clinical Practice." 26 The Three Talk model breaks down the SDM process into three practical steps: (1) Team Talk-engaging the patients and initiating SDM conversation and making sure that patients know that reasonable options are available and a decision needs to be made; (2) Option Talk-providing patients the best available evidence and more detailed information about options. This is often best done outside the clinic visit encounter since patients want time to study new information, consider their personal preferences, and discuss with others; and (3) Decision Talk-considering the different options and preferences and making a decision regarding the best option together with their provider during the clinical encounter. ${ }^{7,27}$ The SDM process provides patients with the support they need to make the best individualized care decisions.

In this study, we will leverage the VA's robust patient portal, MHV. Besides allowing Veterans secure online internet access to their medical records, it gives them ability to communicate via SM with their health care team. To use MHV, Veterans register online and "opt in" to use SM, which is akin to a secure e-mail. ${ }^{28}$ Since SDM can be facilitated by preparatory education, most of our intervention focuses around educating patients regarding menopause. We have designed educational activities to support the SDM process using the SM function of MHV for the Choice and Option Talk steps, ultimately with the goal of enabling Decision Talk, or SDM during a face-to-face encounter.

\section{Study participants and setting}

We will offer the intervention to all women in the perimenopausal age group in the Miami VAHS in Florida. The comparison groups comprised women in two nearby VAHS (West Palm Beach and Orlando) who will not receive the intervention, and women from the intervention (Miami) site who use MHV but decline enrollment and agree to complete baseline and follow-up surveys. All three VAHS have $\sim 3000$ women registered to receive primary care, of which approximately half are between the ages of 45 and 60 .

\section{Inclusion criteria and recruitment for MEANS intervention}

Intervention group. Inclusion criteria for potential intervention group participants are women veterans aged 45-60 years who have had $\geq 1$ primary care visit in the previous year at the Miami VAHS, are registered or willing to register for MHV with SM, and are currently using or willing to learn to use SM. 
Study recruitment will include sending introductory letters describing the intervention to all eligible women in the Miami VAHS. Women already using MHV and SM will get SMs in addition to the mailed letter. We will ask women to contact us by SM or by phone if they are interested. Women who express interest will be provided details about the intervention, and offered instructions and assistance with signing up for MHV and SMs by the project coordinator.

Comparison group. Inclusion criteria for comparison group participants are women aged $45-60$ who had $\geq 1$ primary care visit in the previous year at the Miami, West Palm Beach or Orlando VAHS. The comparison group will continue to receive usual care. All eligible women at these three sites will be mailed an introductory letter and questionnaire in a preaddressed stamped envelope at baseline and at 6months. The West Palm Beach and Orlando women will return anonymous questionnaires, while the Miami comparison group will have assigned study ID numbers. Women who respond at each time point comprise the comparison group at baseline and 6-months, respectively. The respondents may be different at each time point and are anonymous, with the exception of the Miami comparison who will be identified.

\section{Intervention}

Educational content. We compiled various VA-approved educational materials on 24 topics addressing menopause symptoms, treatments, and SDM (Table 1), which we converted into 1-2 page PDFs. We tailored content keeping it at a 5-6 grade reading level so it is easy to understand, and suitable for dissemination via MHV. In addition, we selected three online, interactive decision support tools to facilitate SDM on menopause-related topics of osteoporosis and urinary incontinence from the Agency for Healthcare Research and Quality website (https://ahrq.gov/), and a health decisionmaking template from the Ottawa Research Institute of Canada (https://decisionaid.ohri.ca/decguide.html). We incorporated these decision aids as hyperlinks in the educational material since decision aids have been shown to improve SDM and outcomes. ${ }^{8}$
Patient intervention. For this project, we created a virtual "Miami MEANS" clinic. Miami veterans who agree to be part of the project will be enrolled in this virtual clinic by the project coordinator. The MEANS project coordinator will be familiar with the VA, the patient care teams, and care coordination. However, the project coordinator will not need to be a nurse or medical professional. An overview of the SDM steps and corresponding study intervention strategies is shown in Figure 1. The 6-month intervention is described below and includes the follolwing:

1. Weekly messages: Participants will receive a weekly SM with educational factsheets about menopause with a question at the end based on the content. Participants will respond via SM at their convenience.

2. Monthly monitoring of menopause symptoms: Participants will also receive monthly questionnaires regarding menopause symptoms using the validated Women's Health Questionnaire via SM. The project coordinator will monitor participants' responses. The project coordinator will review the messages and respond as best possible, and reassign to patients' primary care team members only if needed, via SM.

3. Secure messaging with the project coordinator: Participants may report menopause symptoms or any concerns or questions via SM. Participant's SMs will be reviewed, replied to, and clarified, as needed, via SM or telephone, within 48-72 hours. Secure messages with patient responses and questionnaire data will only be viewed by the study coordinator, and will not be saved in the patient's medical records, unless a matter needs immediate attention.

\section{Data Collection}

We will query VA data to obtain lists of women at the three VAHS who satisfy the study eligibility criteria. We will abstract the following information: name, age, address, telephone number, and MHV use. A baseline questionnaire will be mailed to all potentially eligible women in the intervention and comparison groups. The baseline questionnaire takes about 30 minutes to complete and will not be compensated.

Table 1. List of Topics Included in the Educational Materials

What is Shared Decision-Making?

Perimenopause

Coping with Perimenopause

Natural treatments of menopause

Bioidentical hormones-sorting Myths from Facts

Major health risks on low estrogen

How hormone therapy helps

Deciding about hormone therapy

Common hormone therapy programs for women

Hormone therapy for women

Deciding about hormone therapy-Risks and Benefits

Hormone therapy may not be for all women-listing contraindications

Menopause and sexuality-treatment options. Who should avoid hormone therapy?
Sleep problems in menopause

Heart disease-what women need to know Menopause and sexuality

Be safe-prevent intimate partner violence

Urinary incontinence

Depression

Menopause and mental health

Osteoporosis

Healthy eating

Reading food labels 


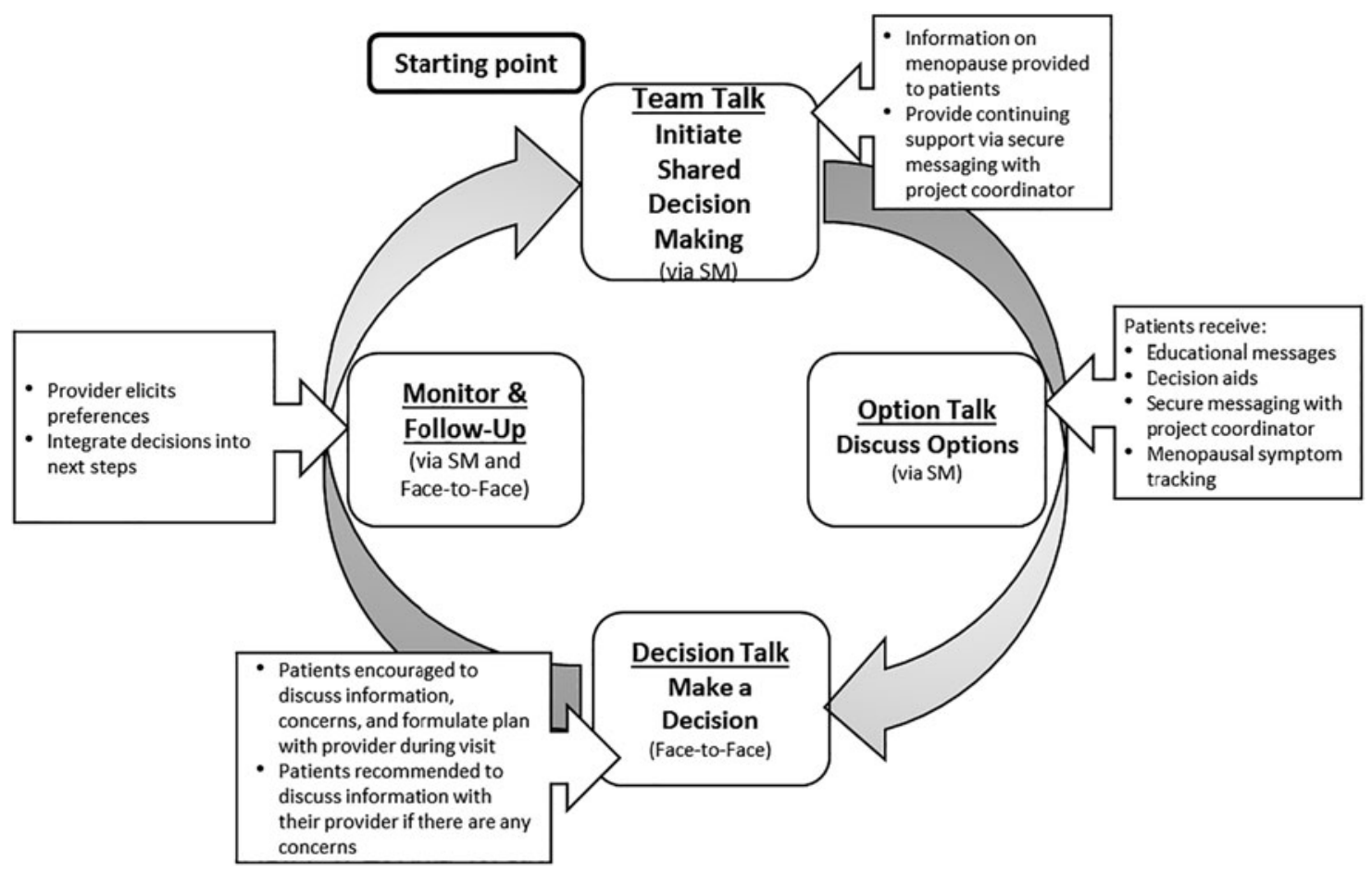

FIG. 1. MEANS underlying framework and overall project intervention: Three Talk Model of Shared Decision-Making Via Electronic Health Record. The team talk and option talk will take place using SM via My HealtheVet, while the decision talk will occur during a face-to-face encounter, but can potentially occur via SM. MEANS, My HealtheVet to Enable And Negotiate for Shared decision-making; SM, secure messages.

The baseline questionnaire will be sent to women in all groups to collect sociodemographic, health and computer literacy, ${ }^{29}$ menopause knowledge and attitude, SDM, and MHV use data. Follow-up questionnaires for the intervention and comparison participants will be collected at 6-months. Besides questionnaires, data will be obtained from the VA Computerized Patient Record System (CPRS) and other VA administrative data.

All eligible women at the two comparison sites will be mailed an introductory letter and unidentified questionnaire to gather data at baseline and 6 months, as shown in Figure 2. Data collected from the intervention site will be linked to study IDs, while data from the comparison sites will be deidentified. Data collected from both intervention and comparison groups will be identical, other than interventionspecific questions and menopause-related QoL (Women's Health Questionnaire $)^{30}$ data, which will be gathered from the intervention group only.

\section{Outcome Measures}

Table 2 gives an overview of the aims and outcome measures, which are described below.

\section{Primary objective}

Knowledge. Menopause knowledge will be measured by a modified version of Noroozi's menopause knowledge questionnaire. Knowledge questions include 18 of the original 26 questions regarding the timing, symptoms, treatments, and complications of menopause. The answers to these questions are true or false, with one point for every right answer. $^{31}$

\section{Secondary objective}

Shared decision-making. We will evaluate the impact of MEANS on rates of SDM and dialogue between the providers and patients regarding menopause and its treatment by surveying patients using the following:

a. CollaboRATE is a 3-item, 10-point scale questionnaire to assess the SDM process. Patients are prompted to think of their last provider visit and rate their SDM experience to the following items: How much effort was made to (1) help you understand your health issues? (2) listen to the things that matter most to you about your health issues? and (3) include what matters most to you in choosing what to do next? Final scores range from 0 to 100 with higher scores indicating more SDM. $^{32}$

b. SDM (SDM-Q-9) is a 9-item questionnaire, using a 6point Likert scale, ranging from 0 -completely disagree to 5-completely agree for a total score of 54 . A higher score indicates a higher likelihood from the patient's perspective that SDM occurred during the clinical visit. $^{3}$

c. Massachusetts General Hospital Menopause Decision Quality Worksheet v2.0, section three "Talking with Healthcare Providers" evaluates if SDM regarding menopause treatment options took place during the visit. ${ }^{34}$ The 5-item questionnaire asks two "yes-no", questions and four questions with answers ranging 




FIG. 2. Modified CONSORT flow diagram.

from "a lot" to "not at all". Participants receive one point for a response of "yes", "a lot", or "some." The total points are divided by the total number of items resulting in scores from $0 \%$ to $100 \%$, with higher scores being more favorable toward SDM.

d. The Stanford Communication with Physicians survey uses three items to evaluate communication between patients and their providers. A lower aggregate means less communication occurred, whereas a higher score indicates more communication with physicians. We will use this scale to determine the patient initiative in being proactive with their providers. ${ }^{35}$

e. Frequency of SDM: At the end of the study period, we will conduct a chart review of the participants' medical record to collect data on SDM. We will conduct a key word search for terms suggesting a discussion of menopause and menopause-associated symptom (e.g., medications for menopause symptoms, medications for menopause symptoms, hormone replacement therapy, hot flashes, osteoporosis). The chart records with key word returns and new menopause-related diagnoses (detailed below) will be reviewed thoroughly for appropriate content and timing that indicate an SDM conversation. We will use these data to validate the participant's self-report of SDM.

\section{Program Evaluation}

We will collect information from patients regarding materials, use of MHV, and satisfaction with the intervention.
1. Material understandability and actionability: The Patient Education Materials Assessment Tool (PEMAT$\mathrm{P}$ ) is a tool from the Agency for Healthcare Research and Quality which systematically evaluates patient education materials and content. It is designed to help determine whether patients will be able to understand and act on provided information. ${ }^{36}$

2. Usability of MHV and SM:

a. Ease of use: This will be assessed using the System Usability Scale (SUS), a 10-item attitude questionnaire using a Likert scale giving a global view of subjective assessment of usability. We will use the SUS after the 6-month MEANS intervention via MHV to determine the participants' opinion on the use and need for technical assistance with MHV. ${ }^{37}$

b. Frequency of use: MHV use will be assessed in the intervention group by the number of participants reading the SMs weekly and sending completed questionnaires back. We will track the SM to monitor the "read status" by utilizing the coordinator view of the SM system. ${ }^{38}$

c. MHV barriers: This study-specific questionnaire will be used to assess MHV ease of use and barriers to consistent use.

3. Program satisfaction: We will use an end of study questionnaire with both Likert scale and open-ended questions. We will obtain detailed information about program quality overall and barriers and facilitators to accessing and using MHV and MEANS resources, such as the pdfs and decision aids. We will use a 
Table 2. ObJectives, Outcome Measures, Tools, and Study Schedule

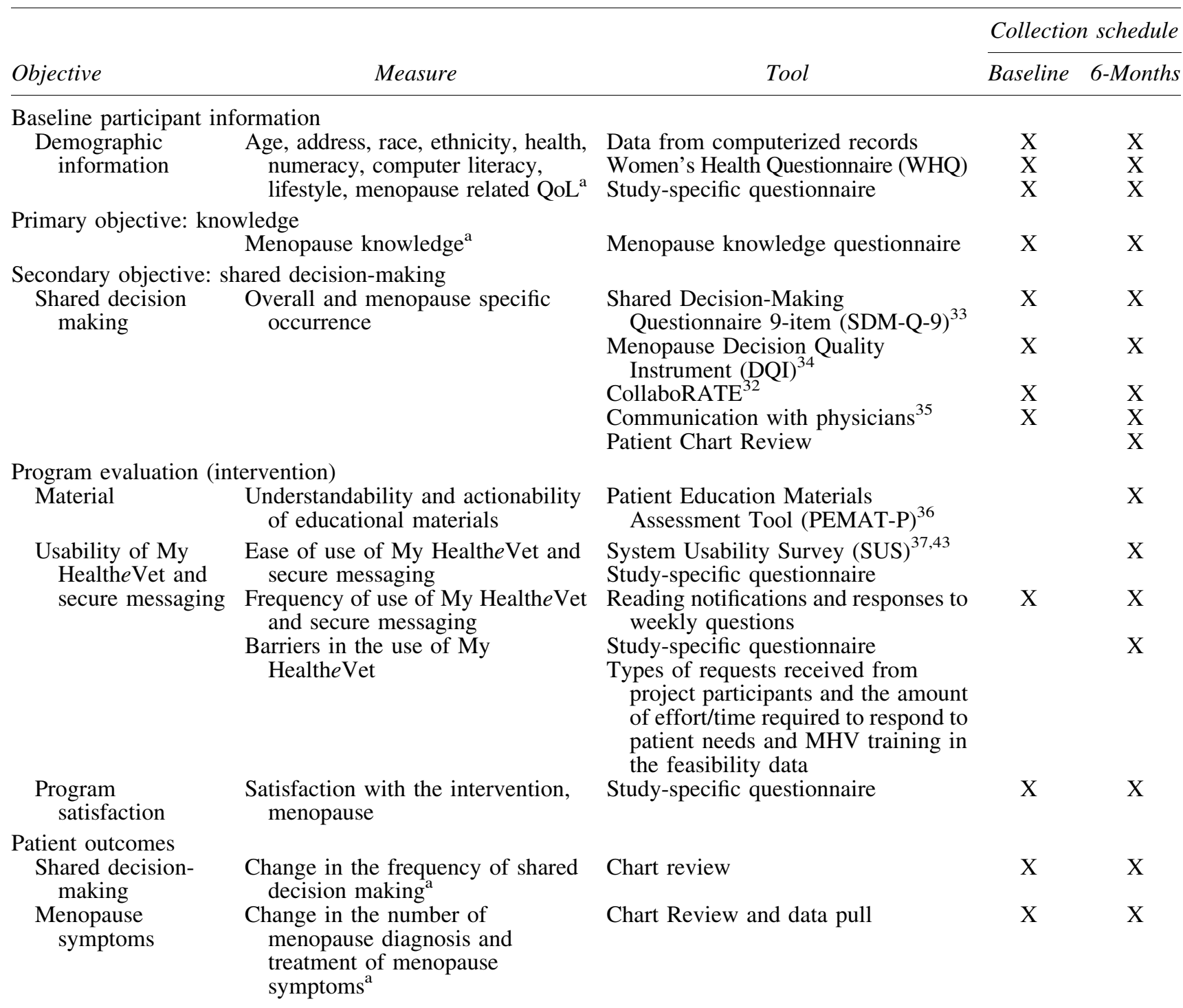

${ }^{a}$ Denotes measures that will also be collected from the comparison group.

QoL, quality of life; MHV, My HealtheVet.

study-specific questionnaire to measure attitudes regarding the use of patient portals for SDM and QoL improvement.

4. Rates of diagnosis and treatment of menopause: We will compare data in the 12-month period pre- and postintervention to determine the number and proportion of women who have an 10th revision of the International Statistical Classification of Diseases and Related Health Problems (ICD-10) code reflecting diagnosis of menopause or menopausal symptoms, or are on an estrogen preparation (oral, dermal, vaginal), or other medications for hot flashes.

5. Document and analyze the types of requests received from project participants and the amount of effort required to respond to patient needs to aid in future implementation of similar educational interventions. Data will be gathered on time spent by the project coordinator on MHV facilitation for both new and previous users of MHV.

\section{Sample size and statistical power}

Sample size consideration and power analyses were performed based on the primary outcome variable of menopause knowledge. Using a two-sided paired $t$-test with a significance level of 0.05 , we estimate that at least 44 participants with complete data in both the intervention and comparison groups would yield $90 \%$ power to detect a difference of $5 \%$ in knowledge scores of women before and after the intervention, with a standard deviation of $10 \% .^{6,11}$ We anticipate a larger recruitment than the required sample because we expect a high level of interest among all eligible women in this population-based intervention. This will allow for potential loss to follow-up, participants who do not read the SM, and multivariate analyses.

\section{Statistical analyses}

The dependent variables in this study are knowledge, SDM occurrence, and patient satisfaction. Before the 
implementation of any statistical tests, we will use descriptive statistics to assess the distributional properties of the different variables and their interrelationships, as well as to determine missing data and detect outliers. $t$-Tests and analysis of variance will be used to compare groups with respect to variables with normal or approximately normal distributions. Mann-Whitney tests and KruskalWallis $\mathrm{H}$ test will be used for comparisons of groups with respect to continuous variables with skewed distributions; paired $t$-tests for analysis of pre- and post-continuous data; chi-square tests for the comparison of independent proportions; and McNemar's tests for the comparison of paired proportions. Statistical test results with a $p$-value $<0.05$ will be considered as statistically significant. Following univariate analysis, generalized linear models will be used for multivariate analysis for each outcome variable (knowledge, SDM, patient satisfaction), adjusting for relevant covariates, such as age, education, income, health literacy, and previous technology use. The multivariate analysis will also enable us to identify variables that are associated with outcome variables (knowledge, SDM, patient satisfaction). Adherence to SM use will also be evaluated using logistic regression models for the intervention group.

\section{Discussion}

Many women are unaware that the symptoms they may be experiencing are related to menopause because of insufficient knowledge and women and their providers often do not discuss menopause treatment. ${ }^{3-5}$ This timely and scalable study seeks to address these challenges by leveraging patient portal technology to support patient education and SDM.

In the United States, women between the ages of 45 and 64 constitute $43 \%$ of all women veterans receiving health care at $\mathrm{VA}$, and this group is the fastest growing group of female users of health care in the VA. ${ }^{39}$ As the VA seeks to better serve this growing group of women, identifying innovative means to care for them is important.

Key strengths of the MEANS educational intervention are the opportune use of patient portals, and a "cluster" type study design using the intervention in one health care system alongside two corresponding health care systems for comparison. This is reasonable because all three VAHS are part of a national health care system, the care is delivered and structured similarly, and they follow the same guidelines, performance measures, and practice patterns. There also is availability of a unified electronic health records in the VA nationally, and processes to pool and sort data for all three VAHS. Furthermore, the numbers of patients in each VAHS are large, as are the number of providers, which assures reasonable balance in both known and unknown baseline factors in patient characteristics and practice patterns. ${ }^{40}$

This trial tests a low-cost intervention via patient portal, which poses little risk to participants. The trial is applied at a "cluster" level, although there is no randomization of clusters in this trial, and therefore has some "pragmatic" features. ${ }^{41} \mathrm{We}$ minimize the inclusion and exclusion criteria and waive the requirement for an informed consent, to improve participation. Moreover, we keep the protocol simple and lessen trial procedures and data-collection requirements and questionnaire burden for the intervention participants. We use mailed questionnaires to avoid the need for study visits. $^{42}$

The large sample size and the selection of multiple comparisons are additional strengths of the study design. We will compare the change from baseline to end of study within and between groups in knowledge scores and SDM occurrence. We will assess the impact of the intervention on patient knowledge and SDM occurrence regarding menopause-related conditions with their provider in their clinic visits, as indications of a successful intervention. Another strength is assessment of outcomes based on chart review of the participants' medical records to collect data on SDM to validate the participant's self-report of SDM, and use of ICD-10 codes for diagnosis of menopause and use of medications for menopause symptoms, independent of self-report.

This study has several limitations. This is a quasiexperimental nonrandomized quality improvement pilot study. The intervention site sample may not be truly representative of the general women population since we will target women who already use MHV or are willing to, while this does not apply to the comparison sites. Since it is well documented that patients who enroll for patient portals tend to be more activated, some group difference may occur by simply having more "engaged" women who are enrolled for MHV in the intervention arm. We will try and accommodate for this by having the Miami comparison group composed of women who are MHV users, therefore the comparison with the Miami control participants will have equally "engaged" women in both groups. Moreover, we will gather data in both groups at two time points and analyze between- and within-group differences to account for these limitations. One of the biggest limitations is that the comparison group participants from the other locations are deidentified, thus making it mostly a comparison of populations, and not individuals. However, comparison participants from the Miami site will be identified and compared at patient level.

Another limitation is that patient communication related to menopause questions will not be saved in CPRS, except patient communication that required consults or primary care intervention, wherein the provider will be added as a cosigner. Therefore, this project will only capture the impact of the intervention on patient behavior and patient-initiated SDM, and does not account for the potential impact of providers seeing menopause-related messages in the patient record, and the impact of that on patient-provider communication around perimenopausal issues. That will remain to be explored as an important part of the patient portal intervention in the future. Yet, another limitation is that we will assess patient perception of SDM, but will not evaluate provider perceptions.

To our knowledge, MEANS is among the first interventions to assess use of a patient portal to promote SDM conversation between patients and providers regarding menopause. It addresses barriers posed by programs that use a face-to-face approach for education of perimenopausal and menopausal women. If successful, our program will add a scalable and timely modality to promote SDM for managing nonacute conditions such as menopause. 
The present proof of concept study is especially important since such interventions for chronic conditions via patient portals would be helpful to all health care systems, especially with the widespread implementation and required "meaningful use" of electronic health records mandated by the Health Information Technology for Economic and Clinical Health act. ${ }^{17}$ Further analyses will allow for evaluation of the effectiveness of the program and population health impact of this approach. Sustained efforts into careful use of patient portals are needed to harness their potential for promoting high-quality conversations between patients and providers.

\section{Acknowledgments}

The authors gratefully acknowledge the support of the Geriatric Research, Education, and Clinical Center (GRECC), Bruce W. Carter Department of Veterans Affairs Medical Center, Miami, FL, and the Miami VA Research. They also thank Alyce Kuo and Avi Lamba for their help with the manuscript. The research reported in this article was supported, in part, by funding from the Pfizer Independent Grants for Learning and Change, Grant ID: 15911255 and by the Miami VA Health care System Geriatric Research, Education, and Clinical Center (GRECC). The investigators retained full independence in the conduct of this research. The views expressed in this article are those of the authors and do not necessarily reflect the position or policy of the Department of Veterans Affairs or the United States government. This quality improvement project's registration number is ClinicalTrials.gov ID: NCT03109145.

\section{Author Contribution}

S.D.: concept, design, analysis, interpretation, and preparation of article. B.T.: conduct, data collection, interpretation, and preparation of article. D.R.: analysis, interpretation, and preparation of article. C.G.-O.: data management and interpretation of VA data. O.G.-M.: design, analysis and interpretation of data, and article preparation. S.L.: study design and article preparation.

\section{Author Disclosure Statement}

No competing financial interests exist.

\section{References}

1. Katon JG, Gray KE, Gerber MR, et al. Vasomotor symptoms and quality of life among veteran and non-veteran postmenopausal women. Gerontologist 2016;56 Suppl 1: S40-S53.

2. Avis NE, Crawford SL, Greendale G, et al. Duration of menopausal vasomotor symptoms over the menopause transition. JAMA Intern Med 2015;175:531-539.

3. Williams RE, Kalilani L, DiBenedetti DB, Zhou X, Fehnel SE, Clark RV. Healthcare seeking and treatment for menopausal symptoms in the United States. Maturitas 2007;58: 348-358.

4. Appling SE, Allen JK, Van Zandt S, Olsen S, Brager R, Hallerdin J. Knowledge of menopause and hormone replacement therapy use in low-income urban women. J Womens Health Gend Based Med 2000;9:57-64.
5. Clinkingbeard C, Minton BA, Davis J, McDermott K. Women's knowledge about menopause, hormone replacement therapy (HRT), and interactions with healthcare providers: An exploratory study. J Womens Health Gend Based Med 1999;8:1097-1102.

6. Saver BG, Gustafson D, Taylor TR, et al. A tale of two studies: The importance of setting, subjects and context in two randomized, controlled trials of a web-based decision support for perimenopausal and postmenopausal health decisions. Patient Educ Couns 2007;66:211-222.

7. Elwyn G, Frosch D, Thomson R, et al. Shared decision making: A model for clinical practice. J Gen Intern Med 2012;27:1361-1367.

8. Stacey D, Legare F, Col NF, et al. Decision aids for people facing health treatment or screening decisions. Cochrane Database Syst Rev 2014:CD001431.

9. Dietz NA, Mijares-Cantrell T, Acevedo D, et al. Women veterans and menopause: Knowledge and preferences. Women Health 2018;58:898-914.

10. Resurreccion DM, Motrico E, Rigabert A, et al. Barriers for nonparticipation and dropout of women in cardiac rehabilitation programs: A systematic review. J Womens Health (Larchmt) 2017;26:849-859.

11. Trudeau KJ, Ainscough JL, Trant M, Starker J, Cousineau TM. Identifying the educational needs of menopausal women: A feasibility study. Womens Health Issues 2011; 21:145-152.

12. What is a patient portal? The Office of the National Coordinator for Health Information Technology (ONC), 2017. Available at: https://healthit.gov/faq/what-patient-portal Accessed January 1, 2018.

13. Vydra TP, Cuaresma E, Kretovics M, Bose-Brill S. Diffusion and use of tethered personal health records in primary care. Perspect Health Inf Manag 2015;12:1c.

14. Dang S, Siddharthan K, Ruiz DI, Gomez-Orozco CA, Rodriguez R, Gomez-Marin O. Evaluating an electronic health record intervention for management of heart failure among veterans. Telemed J E Health 2018 [Epub ahead of print]; DOI: $10.1089 / \mathrm{tmj} .2017 .0307$.

15. Kuo A, Dang S. Secure messaging in electronic health records and its impact on diabetes clinical outcomes: A systematic review. Telemed J E Health 2016;22:769-777.

16. Davis S, Roudsari A, Raworth R, Courtney KL, MacKay L. Shared decision-making using personal health record technology: A scoping review at the crossroads. J Am Med Inform Assoc 2017;24:857-866.

17. Liebovitz D. Meaningful EHR attributes for an era of accountability, transparency, shared decision making, and value assessment. J Leg Med 2013;34:43-53.

18. Kuo AM, Thavalathil B, Elwyn G, Nemeth Z, Dang S. The promise of electronic health records to promote shared decision making: A narrative review and a look ahead. Med Decis Making 2018;38:1040-1045.

19. Grant RW, Wald JS, Schnipper JL, et al. Practice-linked online personal health records for type 2 diabetes mellitus: A randomized controlled trial. Arch Intern Med 2008;168: 1776-1782.

20. Mann DM, Lin JJ. Increasing efficacy of primary carebased counseling for diabetes prevention: Rationale and design of the ADAPT (Avoiding Diabetes Thru Action Plan Targeting) trial. Implement Sci 2012;7:6.

21. Chrimes D, Kitos NR, Kushniruk A, Mann DM. Usability testing of Avoiding Diabetes Thru Action Plan Targeting (ADAPT) decision support for integrating care-based 
counseling of pre-diabetes in an electronic health record. Int J Med Inform 2014;83:636-647.

22. Mann DM, Palmisano J, Lin JJ. A pilot randomized trial of technology-assisted goal setting to improve physical activity among primary care patients with prediabetes. Prev Med Rep 2016;4:107-112.

23. Fiks AG, Mayne SL, Karavite DJ, et al. Parent-reported outcomes of a shared decision-making portal in asthma: A practice-based RCT. Pediatrics 2015;135:e965-e973.

24. Smallwood AJ, Schapira MM, Fedders M, Neuner JM. A pilot randomized controlled trial of a decision aid with tailored fracture risk tool delivered via a patient portal. Osteoporos Int 2017;28:567-576.

25. Krist AH, Woolf SH, Hochheimer C, et al. Harnessing information technology to inform patients facing routine decisions: Cancer screening as a test case. Ann Fam Med 2017;15:217-224.

26. Elwyn G, Durand MA, Song J, et al. A three-talk model for shared decision making: Multistage consultation process. BMJ 2017;359:j4891.

27. Elwyn G, Laitner S, Coulter A, Walker E, Watson P, Thomson R. Implementing shared decision making in the NHS. BMJ 2010;341:c5146.

28. Haun JN, Lind JD, Shimada SL, et al. Evaluating user experiences of the secure messaging tool on the Veterans Affairs' patient portal system. J Med Internet Res 2014;16: e75.

29. Lin X, Wang M, Zuo Y, et al. Health literacy, computer skills and quality of patient-physician communication in Chinese patients with cataract. PLoS One 2014;9:e107615.

30. Hunter MS. The Women's Health Questionnaire (WHQ): Frequently asked questions (FAQ). Health Qual Life Outcomes 2003;1:41.

31. Noroozi E, Dolatabadi NK, Eslami AA, Hassanzadeh A, Davari S. Knowledge and attitude toward menopause phenomenon among women aged 40-45 years. J Educ Health Promot 2013;2:25.

32. Barr PJ, Forcino RC, Thompson R, et al. Evaluating CollaboRATE in a clinical setting: Analysis of mode effects on scores, response rates and costs of data collection. BMJ Open 2017;7:e014681.

33. Kriston L, Scholl I, Holzel L, Simon D, Loh A, Harter M. The 9-item Shared Decision Making Questionnaire (SDMQ-9). Development and psychometric properties in a primary care sample. Patient Educ Couns 2010;80:94-99.
34. Sepucha KR, Feibelmann S, Abdu WA, et al. Psychometric evaluation of a decision quality instrument for treatment of lumbar herniated disc. Spine (Phila Pa 1976) 2012;37: 1609-1616.

35. Lorig K, Stewart A, Ritter P, Gonzalez V, Lynch J, Laurent D. Outcome measures for health education and other health care interventions. Newbury Park, CA: SAGE Publications, 1996.

36. Shoemaker SJ, Wolf MS, Brach C. Development of the Patient Education Materials Assessment Tool (PEMAT): A new measure of understandability and actionability for print and audiovisual patient information. Patient Educ Couns 2014;96:395-403.

37. Heinonen R, Luoto R, Lindfors P, Nygard CH. Usability and feasibility of mobile phone diaries in an experimental physical exercise study. Telemed J E Health 2012;18:115-119.

38. Eguiluz-Perez G, Garcia-Zapirain B. Comprehensive verticality analysis and web-based rehabilitation system for people with multiple sclerosis with supervised medical monitoring. Biomed Mater Eng 2014;24:3493-3502.

39. VA Utilization Profile FY 2016. US Department of Veteran Affairs, National Center for Veterans Analysis and Statistics, 2018. Available at: http://va.gov/vetdata Accessed January 1, 2018.

40. Donner A, Klar N. Pitfalls of and controversies in cluster randomization trials. Am J Public Health 2004;94:416-422.

41. Kim SY, Miller FG. Informed consent for pragmatic trials - the integrated consent model. N Engl J Med 2014;370: 769-772.

42. Ford I, Norrie J. Pragmatic trials. N Engl J Med 2016;375: 454-463.

43. Lewis JR. Measuring perceived usability: The CSUQ, SUS, and UMUX. Int J Hum Comput Interact 2018;34:11481156.

Address correspondence to: Stuti Dang, MD, MPH Geriatric Research, Education and Clinical Center (11 GRC) Miami Veterans Affairs Healthcare System 1201 NW 16th Street, CLC 207 Miami, FL 33125

E-mail: stuti.dang@va.gov; sdang@med.miami.edu 